\title{
Incidência e caracterização morfológica de Alternaria alternata em sementes de salsa
}

\author{
Franciely Magalhães Barroso ${ }^{1}$, Paulo Henrique Pereira Costa Muniz ${ }^{1}$, Gustavo Henrique \\ Silva Peixoto', Mariany Dalila Milan ${ }^{1}$, Daniel Diego Costa Carvalho ${ }^{1}$
}

${ }^{1}$ Universidade Estadual de Goiás, Campus de Ipameri, Ipameri, Goiás, Brasil. E-mail: fran_magb@ @otmail.com,
paulohenrique.muniz1@gmail.com, gugspeixoto@gmail.com, marianydalila@hotmail.com, daniel.carvalho@ueg.br

Recebido: 23/03/2018; Aceito: 23/11/2018.

\begin{abstract}
RESUMO
A queima de alternaria, cujo agente causal é o fungo Alternaria spp., é uma doença ocorrente em áreas de produção de hortaliças da família Apiaceae, devido, principalmente, ao fato de sua veiculação ser por sementes contaminadas. Neste sentido, a qualidade sanitária e fisiológica de sementes é fundamental para o estabelecimento uniforme das culturas em campo, fator que garante o sucesso da produção. O objetivo deste trabalho foi estimar a incidência e caracterizar o fungo Alternaria ocorrente em sementes de salsa cv. 'Graúda Portuguesa'. A análise de incidência foi realizada pelo método blotter test, empregando-se oito repetições (50 sementes por caixa gerbox). Após 10 dias a $25^{\circ} \mathrm{C}$ e fotoperíodo de $12 \mathrm{~h}$ de luz, a avaliação foi realizada examinando-se individualmente todas as sementes em microscópio estereoscópio para a confecção de lâminas semipermanentes, procedendo-se à remoção de micélio e estruturas fúngicas encontradas sobre as sementes infectadas, para caracterização e identificação da espécie do fungo incidente. Os conídios apresentaram dimensões de 21,3 - 37,6 x 6,2 - 13,9 $\mu \mathrm{m}$ $(28,8 \times 10,9 \mu \mathrm{m})$, formato de pera invertida, com coloração marrom clara e bicos de 4,0 - 13,8 $\mu \mathrm{m}(7,7 \mu \mathrm{m})$, com presença de 1-3 septos longitudinais e 2-4 transversais. Estes marcadores morfológicos levaram à identificação do fungo como A. alternata, com $70 \%$ de incidência.
\end{abstract}

Palavras-chave: horticultura, micologia, patologia de sementes.

\section{Incidence and morphological characterization of Alternaria alternata occurring in parsley seeds}

\begin{abstract}
The alternaria black spot, whose causal agent is the fungus Alternaria spp., is a disease that occurs in the areas of production of vegetables of the family Apiaceae, due to its spread by contaminated seeds. Therefore, sanitary and physiological quality of seeds are fundamental for the uniform establishment of the crops in the field, being one of the factors that ensure success of the production. The objective of this work was to estimate the incidence and characterize the Alternaria fungus occurring in parsley cv. 'Grauda Portuguesa' seeds. The incidence analysis was performed using the blotter test method, employing four replicates (50 seeds per gerbox). After 10 days at $25^{\circ} \mathrm{C}$ and $12 \mathrm{~h}$ photoperiod of light, the evaluation was performed by examining individually all seeds in a stereoscope microscope aiming to make semi-permanent slides moutings, which was performed by removing mycelium and fungal structures found on infected seeds, in order to characterize and identify the incident fungal species. The conidia showed dimensions of 21.3 - 37.6 x $6.2-13.9 \mu \mathrm{m}(28.8 \times 10.9 \mu \mathrm{m})$, inverted pear shape, light brown and beaks of $4.0-13.8 \mu \mathrm{m}(7.7 \mu \mathrm{m})$, with presence of 1-3 longitudinal and 2-4 transverse septa. These morphological markers led to an identification of the fungus as A. alternata, with incidence of $70 \%$.
\end{abstract}

Keywords: horticulture, mycology, seeds pathology. 


\section{Introdução}

A salsa (Petroselinum crispum L.) é uma planta herbácea, pertencente à família Apiaceae, que atinge sua importância pelo emprego comercial como condimento. Além disso, tem sido usada na fabricação de cosméticos e sabão, sendo uma cultura rica em substâncias antioxidantes e vitaminas (Filgueira, 2008; Dorman et al., 2011; Mahmood et al., 2014). Segundo o último censo agropecuário para a cultura no Brasil, realizado em 2006, estimou-se que foram produzidas 29.153 toneladas de salsa no país, em que, deste total, 97\% chegaram ao consumidor final (IBGE, 2006).

A grande maioria das hortaliças é propagada por meio de sementes, exemplo disso, pode-se citar a salsa. Neste contexto, a qualidade, principalmente fisiológica e sanitária, é fundamental para garantir o rápido estabelecimento e uniformidade da cultura em campo, condicionando, desse modo, o sucesso produtivo desta olerícola (Nascimento et al., 2011).

Entretanto, o cultivo desta planta pode apresentar problemas, como, por exemplo, a infestação de patógenos às sementes. A associação patógeno-semente é uma das formas que favorecem a sobrevivência e disseminação destes agentes, já que as sementes constituem um veículo de transmissão de doenças a longas distâncias, aumentando a probabilidade de infecção da plântula, causando doenças no início do ciclo (Machado, 1988). A queima de alternaria constitui uma doença de grandes perdas à cultura da salsa (Töfoli et al., 2015). A incidência do fungo Alternaria spp. em sementes de salsa ocasiona baixa germinação, pois afeta diretamente a qualidade fisiológica da semente devido à presença de micotoxinas produzidas pelo fungo, prejudiciais ao desenvolvimento fisiológico (Pedroso et al., 2010; Jajor et al., 2012).

A identificação de espécies, no caso específico do gênero Alternaria, tem sido feita mediante caracterização micromorfológica das estruturas do fungo, de ocorrência em diversos hospedeiros (Rodrigues et al., 2010). As características mais importantes para a eficiente caracterização e a consequente identificação das espécies são as dimensões dos conídios e dos bicos dos conídios que cada uma apresenta (Simmons et al., 2007; Vasconcelos et al., 2014). Devido aos poucos estudos voltados à identificação morfológica de espécies de Alternaria spp., assim como à incidência e associação desse patógeno a sementes de hortaliças utilizadas no Estado de Goiás, o objetivo desse trabalho foi estimar a incidência e caracterizar o fungo Alternaria spp. ocorrente em sementes de salsa a nível de espécie.

\section{Material e Métodos \\ Análise sanitária das sementes}

O estudo foi conduzido no Laboratório de Fitopatologia da Universidade Estadual de Goiás (UEG),
Câmpus Ipameri, no ano de 2018. A análise sanitária das sementes foi realizada pelo método blotter test, de acordo com as Regras para Análise de Sementes (Brasil/Mapa, 2009). Utilizou-se caixas acrílicas transparentes do tipo "gerbox" (11 x 11 x 3,5 cm), previamente esterilizadas com álcool 70\%; em seguida, foram colocados dois papéis mata-borrão previamente umedecidos com água destilada esterilizada a 2,5 vezes seu peso. Posteriormente, sementes não tratadas de salsa cv. 'Graúda Portuguesa' oriundas do comércio local foram semeadas nas caixas "gerbox". O delineamento utilizado foi o inteiramente casualizado (DIC) com oito repetições (50 sementes "gerbox"-1) (Brasil/Mapa, 2009). Após 10 dias a $25^{\circ} \mathrm{C}$ e fotoperíodo de 12 horas de luz, a avaliação da incidência dos patógenos foi realizada examinando-se individualmente todas as sementes em microscópio estereoscópio, estimando-se o percentual de incidência. Os experimentos foram repetidos visando confirmação dos resultados.

\section{Caracterização micromorfológica}

A identificação do gênero fúngico incidente nas sementes e a caracterização morfológica da espécie foram realizadas mediante a confecção de lâminas semipermanentes contendo o material biológico removido diretamente da semente infestada, utilizando ácido lático como meio de montagem para posteriores observações microscópicas (Muniz et al., 2018). Para a caracterização micromorfológica, realizou-se o registro de imagens dos conídios e de suas estruturas, procedendo a mensuração de 30 medidas para cada componente analisado (Vasconcelos et al., 2014), utilizando o microscópio de luz Leica DM500, com auxílio do programa Las Ez 2.0 (100x). As preparações microscópicas foram depositadas no Laminário Fitopatológico da Universidade Estadual de Goiás (UEG), Câmpus Ipameri.

\section{Análises estatísticas}

Os resultados relativos às medidas das estruturas do fungo foram submetidos à análise de variância, com auxílio do programa SISVAR 5.3 (Ferreira, 2011).

\section{Resultados e Discussão}

O teste de sanidade realizado para as sementes de salsa, em duas ocasiões, demonstrou que a incidência de A. alternata foi de $70 \%$. Em seguida, procedeu-se à etapa de caracterização micromofológica do fungo das sementes infestadas.

Após mensuração dos conídios de Alternaria, verificou-se que estes possuíam dimensões de 21,3 37,6 × 6,2 - 13,9 $\mu \mathrm{m}(28,8 \times 10,9 \mu \mathrm{m})$ e bicos com dimensões de 4,0 - 13,8 $\mu \mathrm{m}(7,7 \mu \mathrm{m})$ (Tabela 1). Os conídios apresentaram-se no formato de pera invertida, 
ovoides ou elipsoides de coloração marrom clara, com a presença de 1-3 septos longitudinais e 2-4 transversais (Figuras 1A e 1B). Além disso, observou-se que os conidióforos eram simples, retos ou curvos, lisos, de um a três septos e com perfuração apical. Os conídios apresentaram-se em longas e ramificadas cadeias. Estas características indicaram que a espécie ocorrente nas sementes de salsa era Alternaria alternata. $\mathrm{Na}$ análise das dimensões de A. alternata, verificou-se que o coeficiente de variação para o comprimento e a largura dos conídios foi de $14,16 \%$ e $15,81 \%$, respectivamente.

As sementes naturalmente infestadas foram totalmente colonizadas pelo patógeno, sendo cobertas por uma colônia de coloração cinza escura, aérea variável em densidade, com produção de conidióforos diretamente sobre as sementes, que se estendia também pelo papel de filtro colocado nas caixas "gerbox", impedindo as sementes de germinarem.
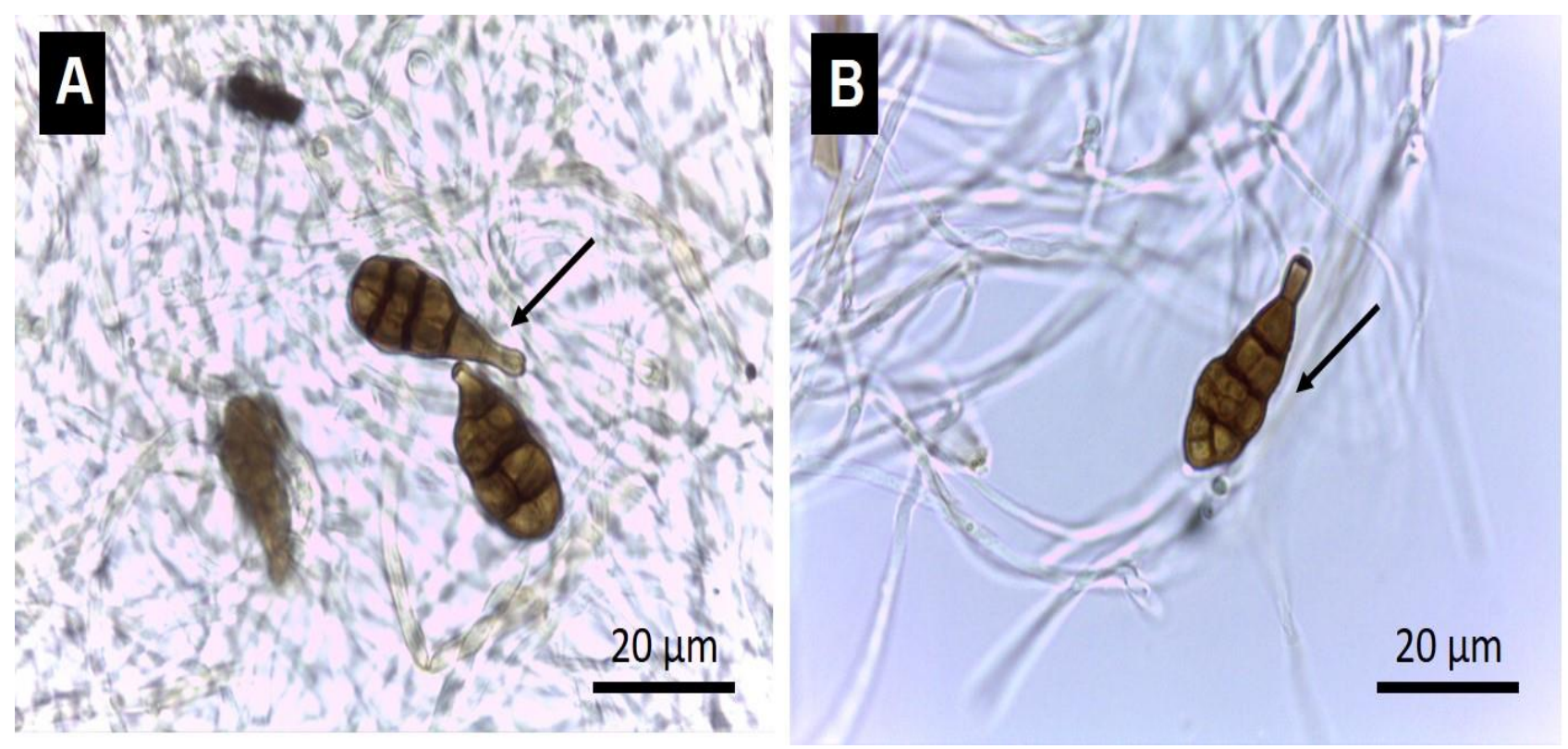

Figura 1. Conídios de Alternaria alternata provenientes de sementes de salsa cv. 'Graúda Portuguesa' (Ipameri, GO, 2018). A) conídios de coloração marrom clara, em forma de clava ou pera invertida, ovóides ou elipsoides. A seta mostra o bico curto, com comprimento inferior a um terço do corpo do conídio; B) a seta mostra o septo transversal do conídio de A. alternata.

Tabela 1. Características morfológicas de diferentes espécies do gênero Alternaria, em diferentes hospedeiros, incluindo Alternaria alternata, encontrada em sementes de salsa cv. 'Graúda Portuguesa' (Ipameri, GO, 2018).

\begin{tabular}{|c|c|c|c|c|}
\hline \multirow{2}{*}{ Espécie de Alternaria } & \multirow{2}{*}{ Hospedeiro / órgão da planta } & \multicolumn{2}{|c|}{ Tamanho do conídio $(\mu \mathrm{m})$} & \multirow{2}{*}{$\frac{\text { Tamanho do bico }(\mu \mathrm{m})}{\text { Comprimento }}$} \\
\hline & & Comprimento & Largura & \\
\hline A. solani $i^{(1)}$ & S. tuberosum / folha & $85,0-100,0$ & $18,0-22,0$ & $83,0-110,0$ \\
\hline A. dauci $i^{(2)}$ & D. carota / sementes & $100,0-450,0$ & $16,0-25,0$ & $5,0-7,0$ \\
\hline A. dauci $i^{(3)}$ & D. carota / folhas & $65,0-115,0$ & $15,0-22,0$ & $*$ \\
\hline A. radicina $^{(2)}$ & D. carota / sementes & $27,0-57,0$ & $9,0-27,0$ & $*$ \\
\hline A. radicina $^{(4)}$ & P. crispum / folhas & 38,0 & 19,0 & $*$ \\
\hline A. alternata $^{(2)}$ & D. carota / sementes & $20,0-63,0$ & $9,0-18,0$ & $2,0-5,0$ \\
\hline A. alternata $^{(3)}$ & D. carota / folha & $25,0-30,0$ & $5,0-9,0$ & * \\
\hline A. alternata $^{(5)}$ & S. tuberosum / folha & $20,0-60,0$ & $9,0-18,0$ & $*$ \\
\hline A. alternata $^{(6)}$ & S. tuberosum / tubérculos & $19,7-45,4$ & $8,5-18,0$ & $2,6-11,9$ \\
\hline A. alternata $^{(7)}$ & P. crispum / sementes & $21,3-37,6$ & $6,2-13,9$ & $4,0-13,82$ \\
\hline
\end{tabular}

${ }^{(1)}$ Rodrigues et al. (2010); ${ }^{(2)}$ Brasil/Mapa (2009); ${ }^{(3)}$ Simmons (2007); ${ }^{(4)}$ Ellis e Holliday (1972); ${ }^{(5)}$ Van der Walls et al. (2011); ${ }^{(6)}$ Vasconcelos et al. (2014); (7)Espécie encontrada em sementes de salsa cv. 'Graúda Portuguesa'. *Não relatado pelos autores. 
Adicionalmente, para as comparações, foram utilizadas as características morfológicas de outras espécies de Alternaria ocorrentes em hospedeiros da mesma família botânica e de A. alternata ocorrentes em olerícolas (Tabela 1). Os resultados das dimensões dos conídios de Alternaria deste trabalho são semelhantes aos que Van der Waals et al. (2011), Vasconcelos et al. (2014), Brasil/Mapa (2009) e Simmons (2007) obtiveram para A. alternata em folhas de batata, tubérculo de batata, sementes e folhas de cenoura, respectivamente. Estes autores encontraram conídios com dimensões de 19,7 - 63,0 x 5,0 - 18,0 $\mu \mathrm{m}$ e comprimento do bico do conídio de 2,5 - 11,9 $\mu \mathrm{m}$ (Tabela 1).

De forma oposta, os resultados das dimensões dos conídios de A. solani e A. radicina (27,0 - 100,0 x 9,0$27,0 \mu \mathrm{m})$ e dos bicos dos conídios $(83,0-110,0 \mu \mathrm{m})$ verificados em folhas de batata por Rodrigues et al. (2010), sementes de cenoura por Brasil/Mapa (2009), e folhas de salsa por Ellis e Holliday (1972), são muito diferentes das medições obtidas pelos conídios de Alternaria em sementes de salsa cv. 'Graúda Portuguesa'. De forma análoga, embora a espécie $A$. dauci seja considerada o agente etiológico da queima de alternaria em salsa, também pode ser refutada, uma vez que seus conídios medem 65,0 - 450,0 x 15,0 - 25,0 e possuem bicos que variam de $5,0-7,0 \mu \mathrm{m}$ (Brasil/Mapa, 2009; Simmons, 2007).

$\mathrm{Na}$ análise das dimensões dos conídios de $A$. alternata, atenção foi dada à variabilidade das medidas obtidas. Assim, verificou-se que o coeficiente de variação, para o comprimento e largura dos conídios, foi, respectivamente, de $14,16 \%$ e $15,81 \%$, diferentemente de A. alternata f. sp. Citri, cuja variabilidade dos dados referentes às medidas de todas as estruturas do fungo não passou de $5,1 \%$ (Carvalho et al., 2008). Estes valores também corroboram com os encontrados por Vasconcelos et al. (2014), que verificaram coeficientes de $23,29 \%$ e $17,82 \%$ para estruturas retiradas diretamente de tubérculos de batata, fato que fundamenta a ideia de que os conídios obtidos diretamente do tecido vegetal do hospedeiro, sem posterior repicagem para meio de cultura, tendem a ser mais variáveis, devido a variações ambientais inexistentes no cultivo in vitro.

Em relação à incidência, A. alternata se mostrou presente em $70 \%$ das sementes de salsa. De forma similar, Nascimento et al. (2006) encontraram para coentro (Coriandrum sativum L.) cv. 'Verdão' um percentual médio de incidência de A. alternata de $95 \%$ em sementes não tratadas. No entanto, Reis et al. (2006) encontraram um percentual médio de $10 \%$ de incidência de A. alternata em sementes de coentro cv. 'Verdão', quando estas foram tratadas com fungicida benomyl. As sementes de salsa cv. 'Graúda Portuguesa' deste trabalho não eram tratadas, o que potencializou grandes percentuais de incidência de A. alternata, que é caracterizada como uma espécie facilmente identificável devido à sua morfologia de conídio e às características da colônia (Casa et al., 2012).

\section{Conclusões}

Baseando-se em marcadores morfológicos, verificou-se a ocorrência de A. alternata em sementes de salsa cv. 'Graúda Portuguesa', com percentual médio incidente de $70 \%$.

\section{Agradecimentos}

Os autores agradecem o apoio oferecido pelo Programa de Bolsa de Incentivo à Pesquisa e Produção Científica (PROBIP) da Universidade Estadual de Goiás (UEG), à Fundação de Amparo à Pesquisa do Estado de Goiás (FAPEG), por uma bolsa de mestrado, e ao Conselho Nacional de Desenvolvimento Científico e Tecnológico (CNPq), pela bolsa de iniciação científica.

\section{Referências Bibliográficas}

Brasil/Mapa. Ministério da Agricultura, Pecuária e Abastecimento., 2009. Regras para análise de sementes. Secretaria de Defesa Agropecuária. Brasília-DF: Mapa/ACS, 399 p.

Carvalho, D.D.C., Alves, E., Batista, T.R.S., Camargos, R.B., Lopes, E.A.G.L., 2008. Comparison of methodologies for conidia production by Alternaria alternata from citrus. Brazilian Journal of Microbiology, 39(4), 792-798.

Casa, R.T., Kuhnem Junior, P.R., Bogo, A., Belani, A.M.M., Bolzan, J.M., Oliveira, F.S., Blum, M.M.C., 2012. Survey, survival and control of Alternaria alternata in wheat seeds. Revista Brasileira de Sementes, 34(3), 358-365.

Dorman, H.J., Lantto, T.A., Raasmaja, A., Hiltunen, R., 2011. Antioxidant, pro-oxidant and cytotoxic properties of parsley. Food \& Function, 2, 328-37.

Ellis, M.B., Holliday, P., 1972. Alternaria radicina. CMI Descriptions of Pathogenic Fungi and Bacteria, 346(1), 1-2.

Ferreira, D.F., 2011. Sisvar: a computer statistical analysis system. Ciência e Agrotecnologia, 35(6), 1039-1042.

Filgueira, F.A.R., 2008. Novo Manual de Olericultura: agrotecnologia moderna na produção e comercialização de hortaliças, third ed. Universidade Federal de Viçosa, Viçosa, $421 \mathrm{p}$.

IBGE. Instituto Brasileiro de Geografia e Estatística., 2006. Censo Agropecuário de 2006. http://www.fao.org/fileadmin/templates/ess/ess_test_folder/W orld_Census_Agriculture/Country_info_2010/Reports/Reports _4/BRA_POR_REP2_2006.pdf. (Accessed: May, 9, 2017). 
Jajor, E., Kozlowska, M., Wojtowicz, M., 2012. Prevalence of fungi of the genus Alternaria on rape siliques and seeds depending on weather conitions. Progress in Plant Protection, 52(4), 1011-1015.

Mahmood, S., Hussain, S., Malik F., 2014. Critique of medicinal conspicuousness of Parsley (Petroselinum crispum): a culinary herb of Mediterranean region. Pakistan Journal of Pharmaceutical Sciences, 27(1), 193-202.

Muniz, P.H.P.C., Marques, M.G., Peixoto, G.H.S., Simão, K. G., Carvalho, D.D.C., 2018. Caracterização morfológica de Alternaria alternata associado a sementes de alface americana cv. 'Astra'. Revista de Agricultura Neotropical, 5(1), 82-86.

Nascimento, W.M., Pereira, R.S., Freitas, R.A., Blumer, L., Muniz, M.F.B., 2006. Colheita e armazenamento de sementes de coentro. Pesquisa Agropecuária Brasileira, 41(12), $1793-$ 1801.

Nascimento, W.M., Dias, D.C.F.S., Silva, P.P., 2011. Qualidade da semente e estabelecimento de plantas de hortaliças no campo. In: NASCIMENTO, W. M. (Ed.). Hortaliças: tecnologia de produção de sementes. Embrapa Hortaliças, Brasília-DF, pp. 79-106.

Pedroso, D.C., Menezes V.O., Muniz, M.F.B., Piveta, G., Tunes, L.M., Muller, J., Menezes, N.L., 2010. Métodos de inoculação de Alternaria alternata e A. dauci em sementes de salsa e sua influência na qualidade fisiológica. Revista Brasileira de Sementes, 32(3), 79-85.

Reis, A., Satelis, J.F., Pereira, R.S., Nascimento, W.M., 2006. Associação de Alternaria dauci e A. alternata com sementes de coentro e eficiência do tratamento químico. Horticultura Brasileira, 24(1), 107-111.

Rodrigues, T.T.M.S., Berbee, M.L., Simmons, E.G., Cardoso, C.R., Reis, A., Maffia, L.A., Mizubuti, E.S.G., 2010. First report of Alternaria tomatophila and A. grandis causing early blight on tomato and potato in Brazil. New Disease Reports, 22(1), 28.

Simmons, E.G., 2007. Alternaria: an identification manual. CBS Biodiversity Series, 6(1), 1-775.

Töfoli, J.G., Domingues, R.J., Ferrari, J.T., 2015. Alternaria spp. In Oleraceous: Symptons, Etiology, Management and Fungicides. Biológico, 77(1), 21-34.

Van Der Waals, J.E., Pitsi, B.E.; Marais, C.; Wairuri, C. K., 2011. First report of Alternaria alternata causing leaf blight of potatoes in South Africa. Plant Disease, 95(3), 363.

Vasconcelos, C.V., Silva, D.C., Carvalho, D.D.C., 2014. Ocorrência de Alternaria alternata (Fr.:Fr.) Keissl. em tubérculos de batata, no Brasil. Pesquisa Agropecuária Tropical, 44(2), 219-222. 